\title{
Histoire de l'art et anthropologie 5. Actualisation, appropriation, appréhension
}

Dijon, 25 mars 2011

\section{Coordination Eliana Magnani et Daniel Russo}

\section{(2) OpenEdition}

\section{Journals}

Édition électronique

URL : https://journals.openedition.org/cem/12003

DOI : $10.4000 /$ cem.12003

ISSN : 1954-3093

Éditeur

Centre d'études médiévales Saint-Germain d'Auxerre

Édition imprimée

Pagination : 283-304

ISSN : 1623-5770

\section{Référence électronique}

Coordination Eliana Magnani et Daniel Russo, « Histoire de l'art et anthropologie 5. Actualisation, appropriation, appréhension », Bulletin du centre d'études médiévales d'Auxerre | BUCEMA [En ligne], 15 | 2011, mis en ligne le 23 août 2011, consulté le 22 septembre 2022. URL : http:// journals.openedition.org/cem/12003; DOI : https://doi.org/10.4000/cem.12003

Ce document a été généré automatiquement le 22 septembre 2022.

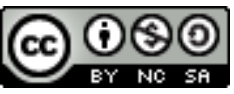

Creative Commons - Attribution - Pas d'Utilisation Commerciale - Partage dans les Mêmes Conditions 4.0 International - CC BY-NC-SA 4.0

https://creativecommons.org/licenses/by-nc-sa/4.0/ 


\section{Histoire de l'art et anthropologie 5. Actualisation, appropriation, appréhension}

Dijon, 25 mars 2011

\section{Coordination Eliana Magnani et Daniel Russo}

Prolongeant la réflexion lancée en 2010 sur la relation " au modèle et à la copie » ${ }^{1}$, et s'insérant pour la cinquième année consécutive dans la réflexion conjointe que nous menons, sur l'art et l'anthropologie, nous nous intéressons, à présent, aux notions «d'actualisation », « d'appropriation » et « d'appréhension », que nous nous proposons de définir, puis de contextualiser dans le champ des sciences humaines et sociales, et d'analyser, à partir d'exemples précis, choisis à la charnière entre anthropologie, histoire et histoire de l'art. Selon notre perspective, il s'agit, d'une part, d'envisager le problème à partir du travail théorique et anthropologique d'Eduardo Viveiros de Castro, sur l'ethos amazonien de la "prédation généralisée " ${ }^{2}$, d'autre part, de comprendre les relations tissées dans l'art médiéval occidental avec le proche, l'Orient, et le lointain, l'antique et/ou le tardo-antique ${ }^{3}$.

Programme

- Eliana MAGNANI, Le Même et l'Autre indispensable, ou comment prendre le socius : autour du travail de Viveiros de Castro

- Angélique FERRAND, L'iconographie du zodiaque, sources de développements par rapport à l'antique

- Raphaël DEMÈs, Iconographie du paon et du phénix : entre tardo-antique et médiéval

- Mathieu BEAUD, La séquence des mages : l'épisode de leur réception par Hérode, autour de l'iconographie du palais, entre antique et médiéval

- Daniel Russo, Les icônes importées de l'Orient à l'Occident? Questions sur une image « métisse » au XIII siècle

3 Tour de table conclusif 


\section{Modèle, copie, un processus}

Présentant l'iconographie du zodiaque, Angélique Ferrands'interroge sur ses sources et ses développements par rapport à l'antique, en reposant la question du "modèle » dans la tradition zodiacale, à travers un aperçu sur l'évolution et la prise en compte du processus à l'œuvre dans l'organisation des images. L'histoire du zodiaque est faite de transferts multiples entre des époques et des cultures différentes, d'où s'ensuivent sa richesse, mais également sa complexité. Ainsi, pouvons-nous parler de «modèle " pour la tradition du zodiaque? Ou plutôt ne devons-nous pas nous demander ce qui " modèle » et ce que « modélisent » les images du zodiaque ${ }^{4}$ ?

Elle distingue, d'abord, les signes du zodiaque, des "fables inventoriantes" et des repères figurés ${ }^{5}$, pour mieux revenir à la question sur le "modèle », que nous pouvons aussi penser dans les termes de la longue tradition. En effet, le zodiaque a des origines mésopotamiennes, mais les douze signes, que nous connaissons aujourd'hui, ne seront néanmoins réellement mis en place qu'au $\mathrm{IV}^{\mathrm{e}}$ siècle avant notre ère et correspondent au découpage des constellations formant le "chemin du soleil» au cours d'une année ${ }^{6}$. Ainsi, les signes du zodiaque vont-ils à la fois devenir des repères dans l'espace, celui du ciel, mais aussi dans le temps, celui de l'année. À travers les écrits initiateurs d'Aratos de Soles ${ }^{7}$ (315-239 av. J.-C.) et Ératosthène ${ }^{8}$ (284-204 av. J.-C.), qui seront commentés, traduits et copiés, les signes du zodiaque font l'objet d'une mythologisation progressive, qui s'avère fondamentale pour la construction iconographique du zodiaque. La mythologie constitue un répertoire ${ }^{9}$, mais elle sert également de déclencheur à tout un processus mnémonique. Autrement dit, elle donne forme aux signes zodiacaux et elle en fait des «fables inventoriantes». Les signes du zodiaque deviennent, en quelque sorte, les «modélisations » du ciel et du temps, et le zodiaque ou le « cercle des figures » forme donc un riche ensemble de repères figurés ${ }^{10}$, dont les formes ne sont pas fixes, mais dont les signes se doivent d'être actifs et identifiables. Le zodiaque participe, entre autres applications, à l'illustration du calendrier dans lequel chacun des signes est associé au mois qui lui correspond, et agit ainsi comme un double relais, visuel et mémoriel ${ }^{11}$. D'autre part, si l'illustration antique du corpus aratéen ne nous est parvenue aujourd'hui que par l'intermédiaire des copies médiévales, forcément tronquées, les occurrences du zodiaque dans d'autres types de documents permettent d'évaluer son iconographie durant l'Antiquité ${ }^{12}$.

6 Pensé sur le mode d'une opération sur l'univers, le zodiaque est aussi conçu comme un résumé du cosmos qu'il ceint de son cercle et il renvoie au concept d'éternité. Ces idées sont rendues accessibles par les formes, ainsi que par l'organisation des images zodiacales ${ }^{13}$, en passant aussi par la mise en réseau visuel des figures. Une imagerie classificatoire est élaborée autour d'une grande figure centrale, en quelque sorte souveraine, dont le zodiaque devient l'attribut de puissance et qui peut être le Soleil/ Helios, Sol Invictus, Aiôn, ou le dieu Mithra, etc. Le tout forme un réseau figuratif ${ }^{14}$, dont nous avons pu constater une certaine récurrence, formé de cercles ou d'arcs concentriques, qui enserrent les signes zodiacaux répartis de manière rayonnante autour d'une figure centrale, le tout inscrit dans un cadre accueillant d'autres unités cosmico-temporelles ${ }^{15}$.

7 Elle note encore que ce type de dispositif figuratif a été repris dans les synagogues entre les $\mathrm{IV}^{\mathrm{e}}$ et $\mathrm{VI}^{\mathrm{e}}$ siècles. Trois pavements, que sont les mosaïques d'Hammath-Tiberias ( $V^{\mathrm{e}}$ siècle), de Sepphoris ( $\mathrm{v}^{\mathrm{e}}$ siècle) et de Beth'Alpha ( $\mathrm{VI}^{\mathrm{e}}$ siècle) ${ }^{16}$, résument l'évolution 
du thème et, surtout, illustrent l'insertion du zodiaque dans un ensemble de tradition judaïque. Le cercle du zodiaque entoure la figure d'Helios, le tout encadré des saisons et formant un registre de composition, où il est associé à des scènes bibliques et à l'arche encadrée de menoroth ainsi que d'objets rituels. Ces synagogues sont installées dans un espace fonctionnant, durant toute la période, comme une zone de contact entre différentes cultures et croyances, ce qui favorisa les échanges et les réactions. Sur ces mosaïques, les détails judaïques semblent soigneusement apposés à l'iconographie, tels des marqueurs identitaires, culturels et religieux, participant à la fabrique d'une " image métisse » ${ }^{17}$. L'ensemble témoigne donc d'un riche processus d'actualisation des formes et des figures, en même temps que de leur appropriation, quand nous comprenons sous ces mots tout le processus décrit.

8 Le zodiaque répond aussi à un grand besoin de compréhension du monde et peut être présenté comme un mode de construction et une mise en discours du cosmos. Les idées développées par l'anthropologue Philippe Descola, quant aux ontologies ${ }^{18}$, c'est-à-dire aux différentes manières d'être au monde, permettent d'appréhender les processus sous-jacents à la figuration, mais non pas de distinguer vraiment des modèles. Pour l'iconographie du zodiaque, et selon ce point de vue discuté, l'ontologie de type analogique serait, plutôt, à retenir. Elle procède, en effet, de la volonté de mise en réseau visuel des images, afin de rendre sensible l'ordre du monde dans lequel les signes zodiacaux agissent tels des repères. Il apparaît davantage pertinent d'avancer les " morphologies des relations $"{ }^{19}$, mises en scène dans l'iconographie zodiacale, que de distinguer des modèles tout prêts, et déjà fixés par ailleurs, donc figés et inactifs. Les images zodiacales sont, au contraire, profondément actives, structurées par des mécanismes visuels créant des résonances et des échos, qui induisent une puissante réflexion sur le monde et sur les connaissances qui s'y rapportent.

9 C'est que le zodiaque supporte la transmission des connaissances universelles ; il est un vecteur de la tradition, entendue selon l'acception la plus large. Au Moyen Âge, l'Antiquité est présentée comme l'auctoritas, le modèle ou, mieux, la source des connaissances et le zodiaque tend à être un réceptacle et un médiateur de ce savoir. À travers les écrits d'Isidore de Séville (v. 560-636) ${ }^{20}$,qui initia le genre encyclopédique, le savoir antique et le zodiaque apparaissent comme des moyens d'accès à la connaissance du monde. Les figures mythologiques, qui sont attachées aux signes du zodiaque, sont considérées comme des moyens de compréhension de ce qui est invisible grâce à des repères figurés directement appréhensibles. Ainsi, le zodiaque fonctionne par analogie pour son iconographie, mais aussi par rapport à ce à quoi il renvoie, l'ordre du cosmos.

$10 \mathrm{Au}$ cours du Moyen Âge, le zodiaque fait l'objet d'une certaine reinterpretatio christiana ${ }^{21}$,même s'il nous faut tempérer ce phénomène. D’ailleurs, Eliana Magnani propose de penser ce processus en terme de «transformations ». Le zodiaque est ainsi présent dans des manuscrits non astrologiques, tels que les martyrologes ${ }^{22}$, en tant qu'éléments du calendrier. Nous remarquerons également que le zodiaque est parfois directement mis en correspondance avec des figures, telles que celles des apôtres ou des thématisations chrétiennes ${ }^{23}$.

11 D'autre part, le $\mathrm{xII}^{\mathrm{e}}$ siècle connaît un certain engouement pour une pensée encyclopédique, ce qui va profiter au zodiaque. Il est intéressant de voir qu'en France, les calendriers sculptés à l'époque sont connus dans les régions marquées par l'art antique. Pour la Bourgogne, on trouve le thème du calendrier dans trois grands ensembles monumentaux ainsi que dans une mosaïque de pavement: il s'agit des 
portails de Vézelay, d'Autun, d'Avallon, ainsi que de la mosaïque de pavement de SaintPhilibert de Tournus. Nous retrouvons ponctuellement les signes du zodiaque sur des supports isolés : ainsi, au sein de décors monumentaux, le zodiaque peut être montré en relation avec les occupations des mois, et apparait comme marquant l'impression du temps sur l'espace, en une perspective eschatologique qui s'approprie la tradition antique.

12 Angélique Ferrand en conclut que le zodiaque est à la fois un ensemble de repères figurés dans l'espace, construits comme des « fables inventoriantes » et répondant à un besoin de compréhension du monde transformé en récit, suivant l'ordre du temps inscrit dans l'humain ${ }^{24}$. L'histoire du zodiaque est faite de conflits, de réactions, de rivalités, entre les croyances et les cultures, qui ne se déterminent pas moins les unes les autres avec des répercussions figuratives, dont nous n'avons donné qu'un aperçu. La reprise des analyses de Philippe Descola à propos des ontologies permet de penser, dans le domaine de l'hypothèse, comment les images du zodiaque jouent sur les modes de l'appréhension et de l'appropriation, tout en produisant aussi des manières de voir le monde et de le figurer.

13 Poursuivant l'interrogation sur les rapports avec l'antique, Raphaël Demès envisage alors la renovatio à travers les rinceaux habités et les procédés de continuation/ transformation du «modèle » tardo-antique dans les compositions romaines des $\mathrm{xI}^{\mathrm{e}}$ et XII ${ }^{e}$ siècles. Il considère, de nouveau, la notion de «modèle », en dépassant l'idée d'une simple reprise de schéma faisant autorité pour légitimer une nouvelle iconographie entre l'Antiquité et le Moyen Âge. Les enjeux des emprunts, reprises et reformulations, sont à analyser, particulièrement à Rome, à travers le thème de la renovatio entre les premiers siècles de notre ère et le début du XII ${ }^{e}$ siècle. Afin d'envisager l'éventuel rôle de « modèle ", que pourraient jouer certaines formes de décors tardo-antiques entre les $\mathrm{XI}^{\mathrm{e}}$ et XII ${ }^{\mathrm{e}}$ siècles, il se propose d'observer comment les rinceaux de vigne habités se sont imposés en tant que puissant motif classique, réactualisé à différentes reprises au cours des siècles. En effet, en réfléchissant sur les enjeux d'un éventuel "modèle" iconographique dionysiaque dans l'art paléochrétien, entre les $\mathrm{III}^{\mathrm{e}}$ et $\mathrm{VI}^{\mathrm{e}}$ siècles, il constate que les rinceaux habités vont se transformer progressivement en un arbre de vie dans l'iconographie méditerranéenne, notamment sur les mosaïques de pavement et dans la sculpture. Il rappelle aussi que la portée eschatologique des rinceaux habités, et plus généralement de la vigne, a déjà été développée dans la sphère funéraire antique, selon la perspective du triomphe sur le temps, cette idée ayant été mise en accord avec celles de l'immortalité et de la résurrection. La dimension eschatologique est, de fait, inhérente à la figure de Dionysos, mais également au récipient destiné à recevoir le vin, le canthare, figuré comme la source des rinceaux habités sur certaines mosaïques tardo-antiques ${ }^{25}$. Le canthare et le raisin sont d'abord repris dans l'iconographie catacombale, dès le $\mathrm{III}^{\mathrm{e}}$ siècle, à Rome, mais c'est dans l'art ravennate du $\mathrm{VI}^{\mathrm{e}}$ siècle que l'arbre de vie se déploie, notamment à San Vitale (fig.1). Le cas de Ravenne est intéressant pour considérer la notion de "modèle ", puisque l'art de cette ville, au $\mathrm{VI}^{\mathrm{e}}$ siècle, permet d'étudier une iconographie singulière à mi-chemin entre l'Orient et l'Occident. 


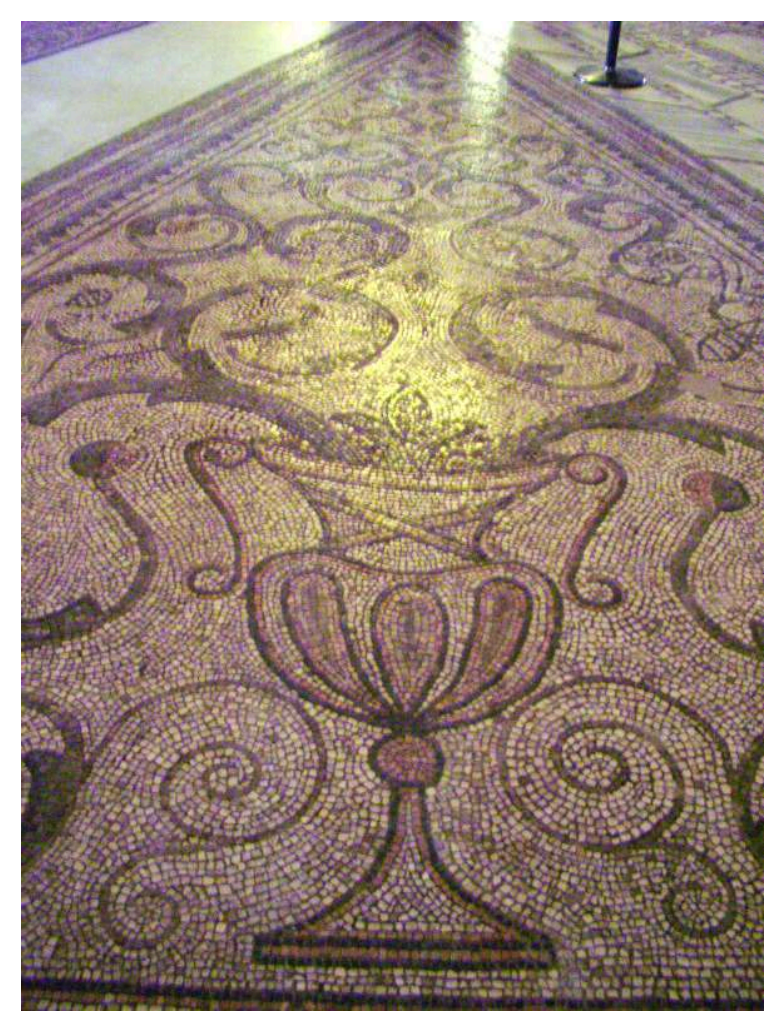

Fig. 1 - Ravenne, basilique San Vitale, segment occidental dans l'axe du presbytère, mosaïque de pavement, Arbre de vie jaillissant d'un canthare, milieu vi ${ }^{e}$ siècle.

Raphaël Demès évalue les enjeux de la reprise des rinceaux habités dans l'espace ecclésial oriental au $\mathrm{VI}^{\mathrm{e}}$ siècle, ainsi que ceux de la transformation progressive du canthare dionysiaque en calice eucharistique. Rachel Hachlili a pu démontrer, pour sa part, que cette iconographie est "populaire » durant toute cette époque en Orient, autant dans l'espace religieux que dans les sphères profane et privée ${ }^{26}$. Il pourrait s'agir de la reprise du motif des guirlandes de vigne hellénistiques, déjà bien effective dans l'iconographie romaine des mosaïques d'Afrique du Nord, entre les $\mathrm{II}^{\mathrm{e}}$ et $\mathrm{III}^{\mathrm{e}}$ siècles. En effet, les réflexions de Michael Avi-Yonah portent à croire que cette composition s'est développée durant le $\mathrm{VI}^{\mathrm{e}}$ siècle en Afrique du Nord, en Italie et en Palestine, au cours de la "renaissance justinienne », ce qui s'accorderait avec la signification de la vigne, et avec la renovatio au programme de Justinien (527-565) ${ }^{27}$. La vigne est un thème qui migra facilement du paganisme vers le christianisme, puisque certains passages évangéliques comparent le Christ à la vigne et ses disciples aux sarments ${ }^{28}$. L'arbre de vie apparaît dans la Genèse, mais peut renvoyer à des strates de significations plus implicites, notamment chez Hippolyte de Rome (170-235) ${ }^{29}$.

Les mosaïques du mausolée de sainte Constance seraient, par ailleurs, un type transitoire pour l'iconographie des rinceaux habités entre le $\mathrm{III}^{\mathrm{e}}$ et le $\mathrm{VI}^{\mathrm{e}}$ siècle. Une section des mosaïques de cet édifice est recouverte de rinceaux habités, réunis au centre de la composition autour du portrait supposé de la défunte. Les différentes étapes du traitement du raisin permettent de considérer cette scène idéalisée comme une reprise d'un répertoire iconographique païen, mais encore comme le manifeste d'une idéologie politico-religieuse développée par le père de la défunte, l'empereur Constantin (312-337) ${ }^{30}$. Le triomphe sur le temps et sur la mort n'est que le reflet d'une volonté de renovatio de l'ancien ordre des choses et des hommes, dans l'Empire romain, 
hautement proclamé par le premier empereur converti au christianisme. Ce dernier affiche la volonté de créer une architecture et une iconographie, dynastiques, en réactualisant les éléments classiques les plus porteurs de sens dans la mise en discours de ses idées impériales ${ }^{31}$.

Ainsi l'iconographie des rinceaux est-elle déjà marquée par une dimension idéologique, dès le milieu $\mathrm{du} \mathrm{IV}^{\mathrm{e}}$ siècle, à Rome, mais la renovatio va également servir un autre discours, celui des réformateurs de l'Église entre les $\mathrm{XI}^{\mathrm{e}}$ et XII ${ }^{\mathrm{e}}$ siècles. Cette hypothèse de travail est examinée à travers trois exemples, à commencer par un sarcophage attique daté du début du III ${ }^{\mathrm{e}}$ siècle, remployé comme sépulture par le pape Damase II en 1048 (fig. 2) ${ }^{32}$.

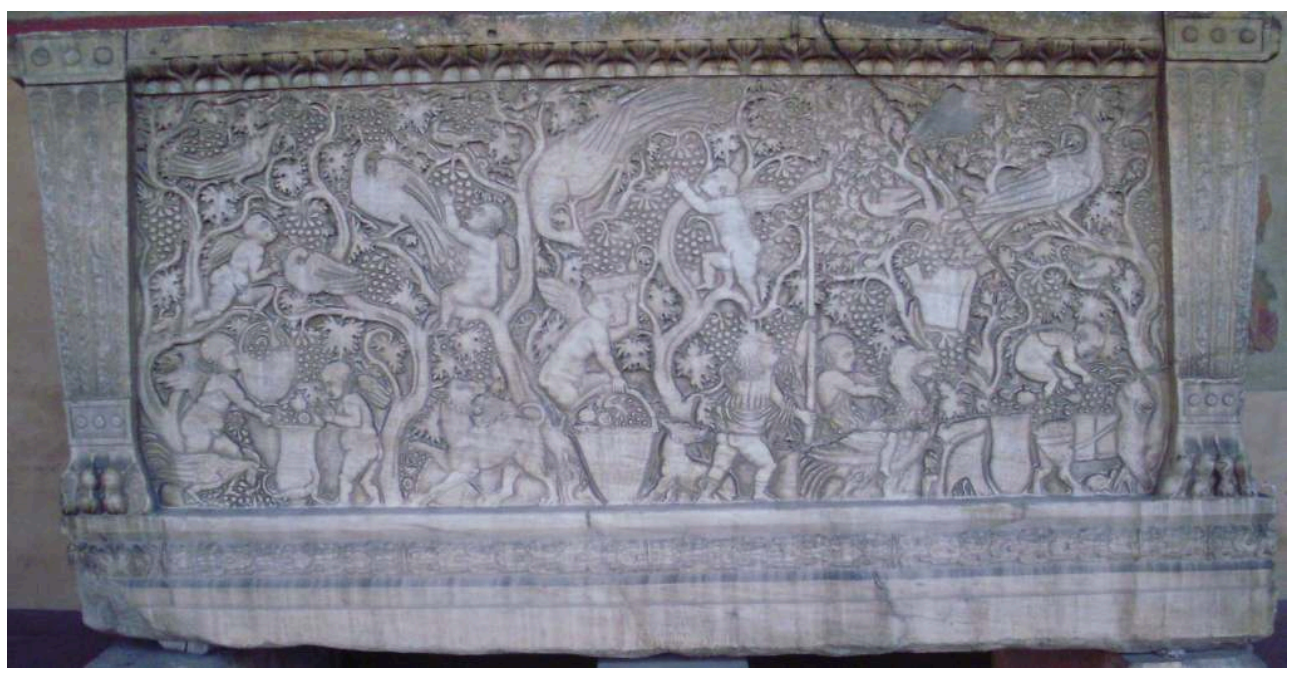

Fig. 2 - Rome, basilique Saint-Laurent-hors-les-Murs, vestibule, sarcophage attique, face avant, Scène de vendanges paradisiaques, début III siècle ; remployé par Damase II en 1048.

La notion de « modèle » est interrogée grâce au remploi à des fins idéologiques, puisque l'iconographie dionysiaque déployée sur ce sarcophage s'accorde avec le discours du pape. Les circonstances de l'accession au trône pontifical de Damase II ont nécessité que ce dernier cherche à légitimer, à inscrire visuellement sa présence à Rome à travers le remploi d'un objet antique afin d'affirmer une emprise spatiale, temporelle et spirituelle, durant son court pontificat. La renovatio véhiculée par les rinceaux habités s'adapte à la portée eschatologique de l'objet, mais également à un contexte pontifical propre au mouvement de renovatio Ecclesiae primitivae formae. L'autre exemple est celui de l'autel de l'église paroissiale de Santa Galla in Circonvallazione Ostiense de Rome, qui est également un remploi d'une ara funéraire antique ornée de rinceaux habités flaviens, donc datés du $\mathrm{I}^{\mathrm{er}}$ siècle de notre ère ${ }^{33}$. Deux textes gravés sur cet autel nous informent, d'une part, que l'autel a été consacré par Grégoire VII - pape de 1073 à 1085 -, le 7 juillet 1073, et, que, d'autre part, l'objet contient désormais des reliques. La deuxième inscription se déploie au centre des rinceaux habités sur la face antérieure de l'autel et se trouve être un palimpseste révélateur d'un nouveau discours. La volonté réformatrice émanant des hautes sphères ecclésiastiques est également rendue sensible par le choix du module graphique, ici l'écriture "grégorienne » du second texte, qui reprend notamment les codes de l'écriture livresque contemporaine, particulièrement l'écriture d'apparat développée dans les Bibles atlantiques. Les rinceaux d'acanthe habités reflètent également l'entreprise ecclésiologique de Grégoire VII marquant sa première année de pontificat ${ }^{34}$. En effet, certains motifs antiques ont retenu l'attention 
des réformateurs, puisqu'ils ont su les appliquer à la littérature monastique contemporaine, notamment aux écrits de Bruno de Segni (1045-1123) et de Pierre Damien (1007-1072) ${ }^{35}$. Les animaux occupant cette végétation sont particulièrement significatifs, dans ce contexte, tout comme l'est l'arbre de vie occupant la face arrière de l'autel.

Cet exemple conduit à réfléchir brièvement sur les mosaïques de l'abside de San Clemente (1118) de Rome, en tant que "manifeste de l'art réformé ». La végétation jaillissant de deux canthares dans la demi-couronne de l'abside se sert de la renovatio de l'iconographie dionysiaque pour composer un nouveau discours. L'art paléochrétien est réactualisé, les figures des apôtres et des martyrs sont exposées, ce qui permet à l'Église, en cours de réforme, de revendiquer ses racines, de légitimer la primauté du pouvoir spirituel sur le pouvoir temporel, de véhiculer enfin toute une ecclésiologie par le moyen de ces mosaïques disposées dans l'espace des églises (des bâtiments). Le schéma de l'arbre serait issu d'un système mnémotechnique en vigueur dans l'enseignement scolastique d'alors, tout comme la crucifixion apparaît comme une synthèse visuelle de la narration pour les clercs ${ }^{36}$. La vigne, en tant que référence à l'Église, les oiseaux et leur vol, en tant qu'exempla des pratiques monastiques contemplatives, sont des thèmes bien représentés sur ces mosaïques, mais aussi dans les écrits de Bruno de Segni ${ }^{37}$ et de Pierre Damien. En effet, le monastère de San Clemente s'inscrit dans un tissu urbain et se trouve donc desservi, à l'époque, par des chanoines séculiers, qui peuvent ainsi profiter de l'impact visuel de leurs prédications sur les fidèles, par le biais d'un discours mis en images. La réappropriation des "modèles » classiques et la réactualisation de l'Antiquité sont particulièrement bien présentes dans l'iconographie romaine, entre le $\mathrm{xI}^{\mathrm{e}}$ et le $\mathrm{xII}$ siècle, d'après les travaux de Stefano Riccioni ${ }^{38}$. La renovatio Ecclesiae primitivae formae passerait donc à travers les emprunts au répertoire antique et paléochrétien, et s'inscrirait dans un vaste mouvement qui ne s'achèverait qu'au début $d u$ xiII siècle, quand le pontificat d'Innocent III aura renouvelé les institutions ecclésiastiques et proclamé l'autonomie politique de l'Église ${ }^{39}$.

Raphaël Demès observe donc que le "modèle» fait autorité par le processus de réappropriation d'une iconographie, antique ou paléochrétienne, ceci étant perceptible à travers le motif des rinceaux habités comme outils visuels de la renovatio. Il a ainsi pu relever une imbrication des niveaux de références, une certaine mise en abîme des modèles successivement étudiés, en passant de l'iconographie dionysiaque à l'art paléochrétien. La végétation luxuriante et harmonieuse est un triomphe sur le temps et, par conséquent, sur la mort. Cette idée est reprise à des fins politiques par Constantin, dès le $\mathrm{IV}^{\mathrm{e}}$ siècle, puisqu'il exploite largement la notion de renovatio dans l'iconographie impériale. Comme en écho, il s'aperçoit en même temps d'une reprise similaire des rinceaux habités à des fins idéologiques par l'Église réformée, au long des $\mathrm{XI}^{\mathrm{e}}$ et $\mathrm{XII}^{\mathrm{e}}$ siècles, cherchant à toujours orienter son discours centré sur le retour aux sources de la primitive Église : la renovatio Ecclesiae primitivae formae (fig. 1 et 2).

Mathieu Beaud étudie, ensuite, «La séquence des mages : l'épisode de leur réception par Hérode ", en s'interrogeant surl'iconographie du palais, entre antique et médiéval, en tenant compte de la pérennité du thème dans le champ d'expression figurée, de son ancienneté et de la stabilité de la structure, tout au long des siècles. Pour mettre en évidence les différences fondamentales, qui existent entre des images distantes de près de dix siècles, la mise en parallèle du thème développé dans la sculpture du XII siècle, 
avec les aboutissements qu'il a connus dans l'art funéraire de l'antiquité tardive, apporte au sujet des éléments de réponses intéressants. La frise inférieure de la façade occidentale de Saint-Trophime d'Arles (fig. 3 et 4 ) se réfère volontairement à ce modèle tardo-antique et offre, pour cela, un excellent terrain d'étude ${ }^{40}$.

21 La sculpture de sarcophage de la période post-constantinienne, par exemple le Sarcophage des Époux, conservé au musée de l'Arles antique, présente un premier stade d'aboutissement du sujet. Les mages sont trois et portent l'or, la myrrhe et l'encens, clairement identifiables. Toutes les références vétérotestamentaires, qui leur sont habituellement associées, sont présentes. Balaam, derrière le trône de la Vierge, montre l'étoile $(\mathrm{Nb} 24,17)$, les chameaux prédits par Isaïe $(I s 60,6)$ accompagnent les mages. L'image respecte les commentaires patristiques, qui tentaient de mettre en évidence les prophéties messianiques relatives à l'épisode. À Saint-Trophime, la cellule iconographique s'est simplifiée. Pourtant, les signes faisant la richesse des décors antiques n'ont pas disparu. Ils sont devenus intrinsèques au thème. Les références messianiques sont omniprésentes dans toutes les productions relatives à l'Épiphanie, dans les commentaires sur les Écritures, les sermons, mais plus intéressant encore, dans toutes les productions sérielles, telles que la glose et les tropes liturgiques. Dans un procédé d'englobement iconographique, l'image médiévale a absorbé l'ensemble de ces signes devenus, dès lors, comme partie intégrante du sujet.

Cependant, l'iconographie médiévale ne fait pas que synthétiser les réflexions antérieures. L'analyse dans le temps des deux séquences de l'Adoration des mages et des Mages devant Hérode permet de déceler l'invention médiévale par rapport au modèle. La composition symétrique des deux épisodes se développe sur une structure originelle disposant, en pendant, l'Épiphanie et les Hébreux dans la fournaise (Dn 3, 19-24) ou des Hébreux face à Nabuchodonosor (Dn 3, 13-18). Cette dernière, par contamination iconographique, se transforme en audition des mages face à Hérode ${ }^{41}$. Dans l'empire carolingien, c'est ce modèle qui est repris. Hérode bénéficie alors d'une véritable fascination et tient une place significative dans les images. Pourtant, la réutilisation du modèle structurel ne signifie pas une reprise sémantique. Là où les sculpteurs antiques, en associant les deux épisodes, représentaient la victoire d'un christianisme triomphant (post-constantinien) face à l'aveuglement de ses ennemis (juifs et païens), les arts précieux germaniques mettent en opposition des sièges de pouvoir. Dans une initiale enluminée de l'Évangéliaire de Drogon (844-855) ${ }^{42}$, la Vierge et Hérode sont disposés sur un même axe vertical, chacun accueillant les mages, et sont séparés par deux petits édicules : Jérusalem et Bethléem. Sur le plat de reliure de l'Évangéliaire de Lorsch $(810)^{43}$, Hérode et la Vierge à l'enfant siègent chacun sur une maquette des villes en question. Enfin, une cassette en ivoire conservée au Louvre ${ }^{44}$, montre une superposition des deux salles royales, le palais d'Hérode et la crèche monumentalisée. Les images impériales montrent donc une iconographie enrichie par rapport à leur modèle. De l'interprétation exégétique des deux personnages évangéliques dans les décors antiques, Mathieu Beaud observe le passage à une opposition mystique des deux sites de pouvoirs (Jérusalem et Bethléem). Dans les images les plus avancées, le dignitaire et le lieu fusionnent pour construire architecturalement l'interprétation exégétique du personnage. En ce qui concerne la Vierge, l'allégorisme architectural de sa virginité, l'aula pudoris ${ }^{45}$, est rendu manifeste dans le Codex Egberti en une architecture sanctuarisée, dont Marie devient la porte close ${ }^{46}$, devant laquelle se présentent les mages. Dans l'imagerie ottonienne, l'Évangéliaire d'Otton III montre la Vierge enveloppée par ce sanctuaire ${ }^{47}$, enchâssée dans une aula regia, qui englobe cette 
fois les mages en une seule et même cellule iconographique ${ }^{48}$. À l'intérieur de cette salle royale, les mages peuvent devenir des Rois mages. Sur la façade de SaintTrophime, la Vierge à l'enfant et Hérode s'opposent de la même manière. Si le modèle visuel assumé est la sculpture de sarcophage, le sens qu'il apporte au programme iconographique est bien celui issu des réflexions germaniques, à savoir la mise en tension de deux souverains dans leur palais, l'un détenteur d'un pouvoir diabolique, l'autre souverain universel, né dans le sein virginal de Marie. La Vierge Église accueille les nations chrétiennes en la personne des mages ${ }^{49}$. La frise arlésienne, incrustée dans un programme eschatologique, donne une valeur céleste au thème. La Vierge présentant le Christ aux nations devient la porte du ciel ${ }^{50}$, pour donner une valeur ecclésiale collective à l'Épiphanie.

Ainsi, l'étude du thème des mages sur une période longue permet-elle de voir plusieurs moyens de transmission des modèles. Une mutation progressive laisse apparaitre des glissements, synthèses et actualisations iconographiques. Dans le cas de SaintTrophime d'Arles, où la référence est faite à un modèle précis, l'analyse est plus significative puisqu'elle laisse apparaître, d'une part, la compréhension du modèle antique et son interprétation, d'autre part, les réflexions médiévales dont l'image ne peut se passer, même en allant aussi loin que possible dans la copie du modèle. Il en découle une meilleure compréhension du thème iconographique présenté pour être vu (fig. 3 et 4 ).

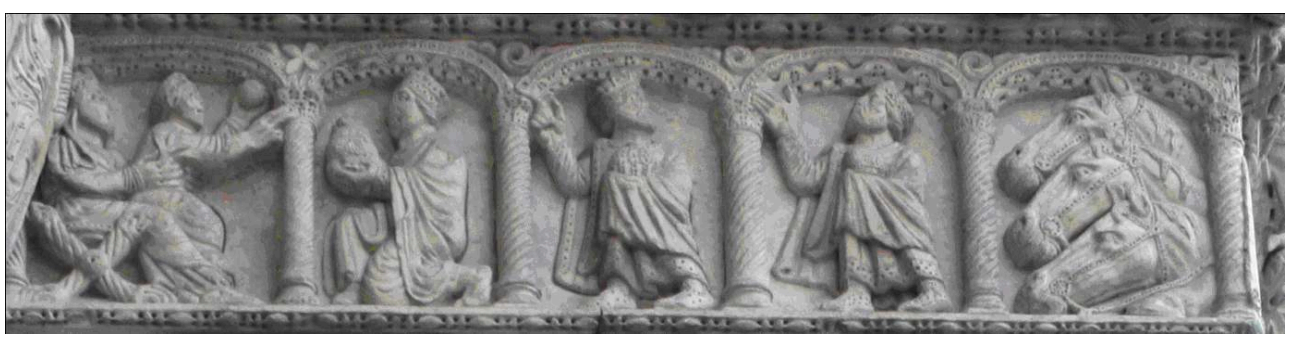

Fig. 3 - Arles, église Saint-Trophime, façade occidentale, frise inférieure, côté gauche, ébrasement sud, Adoration des mages.

Fig. 4 - Arles, église Saint-Trophime, façade occidentale, frise inférieure, ébrasement nord, Les mages devant Hérode.

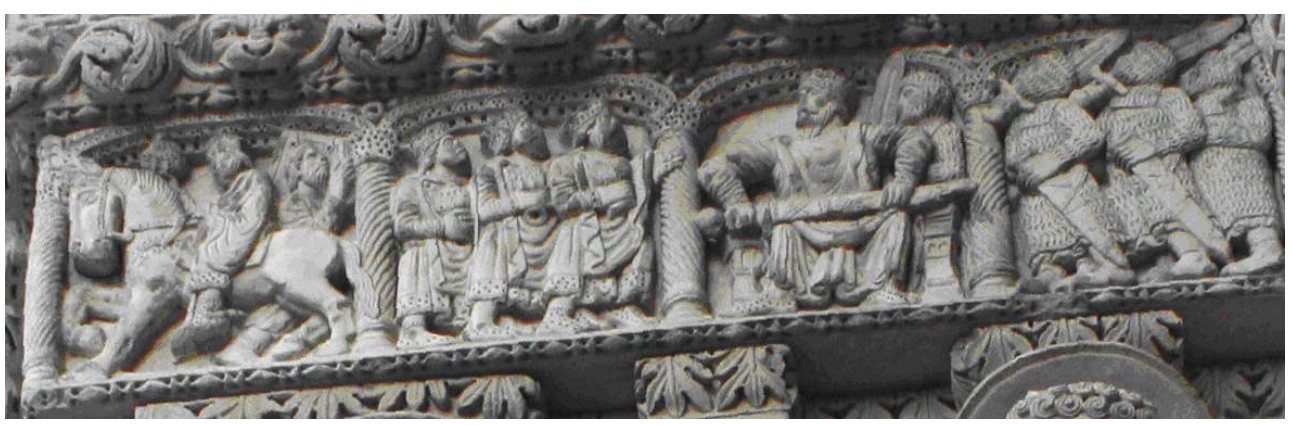

\section{Métissage}

Une ample documentation est disponible et, parfois, rassemblée à la faveur des expositions et des catalogues publiés ${ }^{51}$, et la problématique d'ensemble a été posée, dès 1990, par Hans Belting, dans son ouvrage traduit sous l'intitulé Image et culte, en particulier au chapitre 16 du livre ${ }^{52}$. C'est sous l'angle du métissage que Daniel Russo rouvre, dès lors, le questionnement sur l'importation des icônes en Occident et, plus 
généralement, qu'il pose la question des nouvelles conceptions et des nouvelles techniques de l'image, qui sont, depuis la fin du XI ${ }^{e}$ siècle, grecques ${ }^{53}$. Il concentre son analyse sur les $\mathrm{XII}^{\mathrm{e}}$ et $\mathrm{XIII}^{\mathrm{e}}$ siècles en sachant que le mouvement est, toutefois, bien antérieur, et que les exemples à disposition ne sont que des pièces éparses dans un vaste puzzle, difficile à réunifier et à comprendre. Selon la même perspective, il se propose de substituer aux notions bien éculées - "déplacements d'artistes", "importations» d'objets et d'œuvres dans les deux sens et sur les deux côtés de la Méditerranée, «osmose» entre des milieux «croisés», situations artistiques, (perpétuelle) découverte de l'antique, soit néo-hellénique (du reste), soit néo-latin - un autre ensemble de concepts et de "points de vue»-pour emprunter ce terme à l'anthropologie ${ }^{54}$ : «interculturalité » en lieu et place d' «osmose $»^{55}$; «circulation/ transformation des biens ${ }^{56}$ en lieu et place des "déplacements d'artistes » et des "déplacements d'œuvres "; "métissage » et sa résultante, "hybridation » en lieu et place de la notion de «style» ou, plus vague encore, la notion de «style internationalisé ${ }^{57}$. Ce sont les concepts qui servent à une autre approche des faits observés - actualisation, appréhension et/ou appropriation - entre modèle et copie, sur fond de la circulation des icônes dans le monde méditerranéen et, au-delà, dans toute l'Europe ${ }^{58}$. Actualisation, appréhension, appropriation sont les trois modes privilégiés dans l'étude historique des objets analysés successivement sous l'angle de la documentation d'abord, puis des cas retenus et discutés par Hans Belting, que Daniel Russo examine au cours de son travail.

Le dossier iconographique est, en effet, très complet et connu, pour ses principaux éléments, même s'il ne peut prétendre à l'exhaustivité, bien évidemment. Il comprend, et dans une séquence chronologique homogène, les icônes conservées et toujours visibles pour la plupart d'entre elles, relatives à la tradition de l'icône peinte par l'apôtre Luc, à l'origine d'une série d'autres, réalisées d'après ses principaux traits et diffusant le type de l'Avocate :

- l'icône de la Madone de San Sisto ou Santa Maria del Rosario, après restauration [peinture à l'encaustique originale sur panneau de peuplier ; 71,5/42,5 cm ; avec un fond d'or d'époque ; datée autour $\mathrm{du} \mathrm{VI}^{\mathrm{e}}$ siècle], première icône de la Mère de Dieu considérée, à Rome, vers 1100 , comme ayant été peinte par saint Luc et propriété des religieuses de Santa Maria in Tempuli ${ }^{59}$;

- l'icône de la Sancta Virgo Virginum, Mater Omnium [Venise, collection Cini ; 107/57 cm], icône provenant de San Gregorio Nazianzeno à Rome, en bon état de conservation et entourée de son cadre portant l'inscription Sancta Virgo Virginum ${ }^{60}$;

- l'icône de Santa Maria Nova, sur le forum, à Rome, puis dans Santa Francesca Romana, et qui provenait sans doute de Santa Maria Antiqua, dont le titre et l'inventaire furent transférés sur la nouvelle église mariale au IX ${ }^{\mathrm{e}}$ siècle ${ }^{61}$;

- l'icônede Santa Maria di Grottaferrata, au sud de Rome (bois de cèdre, 125/42 cm), attribuée par Valentino Pace (1985) à un peintre chypriote, qu'Hans Belting présente comme « une icône importée ", " garnie à la fin du siècle [le XIII siècle] par deux volets latéraux "; au $\mathrm{XV}^{\mathrm{e}}$ siècle, « (...) le cardinal Basilius Bessarion (vers 1395-1472) inaugurait la vénération de l'icône comme image de saint Luc ${ }^{62}$ ». Une inflation d'images de ce type se diffusait alors, aux XIII ${ }^{\mathrm{e}}$ puis XIV ${ }^{\mathrm{e}}$ siècles, satisfaisant en quelque sorte le besoin sans cesse plus fort de légitimer le champ visuel marial, en l'associant étroitement au champ d'expression du pouvoir de l'Église romaine, mais aussi universelle. prolifération des variantes, c'est vers l'icône byzantine conservée dans l'église des 
Blachernes, à Constantinople, que le processus renvoie, comme à son archétype et à l'autorité pouvant garantir le passage à l'image ${ }^{63}$. Et dans sa forme, dans le style, dans sa structure aussi, l'image est gréco-latine et se donne à voir comme le lieu d'un métissage. Sa production est ainsi la rencontre interculturelle des pratiques, des savoirfaire et des connaissances. Dès lors, les traits iconiques soulignent, ici et là, ce mixte de composition, aussi bien dans les ateliers situés aux points carrefours entre l'Orient et l'Occident, et tout autant à l'arrivée, dans les communes du nord et du centre de la péninsule italienne : à Spolète, dès les premières années du XII siècle; à Venise, à compter du XIII e siècle, et dans tout son espace de terre ferme, ainsi que sur les côtes de l'Adriatique ; en Ombrie, en Toscane ${ }^{64}$. Daniel Russo ramène ces traits à quelques-uns, fondamentaux, parce qu'ils sont essentiels à la définition de l'icône :

- un très fort ancrage dans le temps même de la production, avec la valorisation du contemporain, parce que la référence à celui-ci est renvoi à l'archétype, à l'archè, en signalant le présent de l'icône comme déjà archétypique, c'est-à-dire proche de l'origine. Les mentions du nom du donateur ou de celui de la donatrice, sur les montants des cadres, refaits à neuf ou simplement rajoutés à frais nouveaux, de même que celles des rappels géographiques ou topographiques jouent toutes en ce sens, en direction d'un présent montré habité par le passé et fondé sur lui ;

- une articulation bien pensée entre le visuel et le textuel, par le biais de l'inscription des lettres écrites en peinture sur le fond de l'icône, sur ses bords, au seuil du modèle figuré, ou encore reportées sur des phylactères de papyrus ou sur des feuillets de parchemins, lettres de suppliques et demandes d'intercession ${ }^{65}$;

- un marquage de propriété avec l'indication - la mise en indice, selon le terme employé par Alfred Gell - du nom du propriétaire, qui reprend l'icône et la fait restaurer, pour mieux l'exposer sur son ancien site - d'ordinaire resté inchangé -, dans l'espace social de la commune urbaine, en Italie surtout, ou l'insérer dans un lieu de dévotion particulier, en milieu de cour notamment ${ }^{66}$. L'icône agit sur des dévotions profondément socialisées, dans les villes d'Italie, mais elle réagit aussi sur une dévotion de plus en plus privée à destination des élites aristocratiques du pouvoir seigneurial, royal ou encore impérial. Et, à ce point d'arrivée, elle peut connaître un autre départ à partir des variantes et des copies qui sont vues et comprises comme les sources du lointain modèle. La conjoncture historique, extérieure à l'image, détermine sa structure interne et renverse les termes des échanges par rapport à l'archétype.

27 Tout en observant la pertinence de l'analyse proposée par Hans Belting, Daniel Russo observe qu'il faut encore travailler, dans une problématique d'étude pourtant bien ouverte, à l'articulation avec l'histoire, et en particulier l'histoire sociale pour la période considérée, et qu'il est en même temps nécessaire d'ouvrir l'icône aux sciences humaines et sociales, pour une compréhension plus assurée des questions posées ${ }^{67}$.

\section{Prédation}

Pour rendre compte et élargir les termes choisis ici pour réfléchir sur la relation entre " modèle » et "copie », c'est-à-dire actualisation, appréhension, appropriation, il a semblé utile à Eliana Magnani de rappeler la force heuristique du décentrement théorique, tel qu'il est proposé par l'anthropologue Eduardo Viveiros de Castro [1951-] à travers les concepts de perspectivisme, de multinaturalisme et d'altérité cannibale, élaborés à partir de son expérience sur le terrain amazonien ${ }^{68}$. Conjuguant 
l'anthropologie symétrique de Bruno Latour [1947-] et la retro-anthropologie (reverse anthropology) de Roy Wagner [1938-], en se fondant sur le structuralisme lévi-straussien et sur la philosophie deleuzienne, Viveiros de Castro lit l'anthropologie européenne à l'aune de l'anthropologie indigène, en soutenant que «toutes les théories anthropologiques non triviales sont des versions des pratiques de connaissance indigène ; ces théories se situent de la sorte dans une stricte continuité structurale avec les pragmatiques intellectuelles des collectifs qui se trouvent historiquement en "position d'objet" au regard de la discipline» $(2009$, p. 6$)$. Son projet théorique vise ainsi à « une reconstitution de l'imagination conceptuelle indigène dans les termes de notre propre imagination [...] de façon à être capables (si tout "va bien") de forcer notre imagination, et ses termes, à émettre des significations complètement autres et inouies » (2002, p. 15). Cette invitation à dialoguer à égalité avec les concepts indigènes - un autre exemple est la lecture du féminisme faite par Marilyn Strathern à partir des concepts mélanésiens ${ }^{69}$ - et de dépasser ainsi la pensée dichotomique occidentale, est d'autant plus éclairante que la façon des peuples amazoniens de voir le monde se trouve être l'exact opposé de celle de nos propres sociétés, dites modernes. Cette approche prend place dans le contexte de multiplication des études sur les indigènes des basses terres de l'Amérique du Sud depuis les années 1970, qui ont vite montré l'insuffisance de la grande dualité nature/culture pour caractériser les cosmologies amérindiennes, une fois que ces collectifs sont constitués de relations entre humains et non-humains dotés de prédicats intrinsèques équivalents ${ }^{70}$. C'est également dans ce creuset des travaux amazonistes qu'ont émergé les propositions théoriques de Philippe Descola, dont Viveiros de Castro est actuellement l'un des principaux critiques ${ }^{71}$.

En ajoutant un troisième terme au paradigme traditionnel de l'anthropologie de la parenté (filiation et alliance), celui de l'affinité virtuelle (ou potentielle) en tant que forme première de socialisation de l'altérité - parentèle distante, affins d'affins non corésidents, affins potentiels, ennemis -, Viveiros de Castro a montré qu'en Amazonie la prédation généralisée, marquée du sceau symbolique du cannibalisme, est la structure englobante, la modalité prototype de la relation. Tout échange est une forme de prédation - entre affins, entre hommes et femmes, entre vivants et morts, entre humains et animaux, entre humains et esprits, entre ennemis. À travers la prédation incorporante, sujet et objet s'interconstituent, dans un mouvement de préhension perspective, où ce qui est incorporé est quelque chose d'éminemment incorporel, la position même de l'autre, son point de vue. Le point de vue crée le sujet; le sujet est celui qui se trouve activé, «agentivé » (au sens d'Alfred Gell) par le point de vue. C'est ce qui définit le perspectivisme amérindien, un monde habité par différentes espèces de sujets ou de personnes, humaines et non-humaines, qui l'appréhendent selon des points de vue distincts. Tous les êtres voient (représentent) le monde de la même façon, ce qui change est le monde qu'ils voient, d'où se dégage le concept de multinaturalisme - ou de "multivers", par opposition au multiculturalisme moderne. Ainsi, la question n'est pas de savoir comment les singes voient le monde, mais quel monde s'exprime à travers les singes, de quel monde les singes sont le point de vue. De même, le perspectivisme n'est pas un relativisme, mais un relationnisme: quelque chose n'est poisson que parce qu'il existe quelqu'un de qui cette chose est poisson. Le point de vue est dans le corps, car si les animaux voient de la même façon que nous des choses diverses de ce que nous voyons - pour nous c'est le sang, pour le jaguar c'est la bière de maïs ; pour l'âme des morts c'est le cadavre pourri, pour nous c'est du manioc; pour nous c'est une flaque de boue, pour les tapirs, c'est la grande maison cérémonielle... -, 
c'est que leurs corps sont différents des nôtres. Un corps est un faisceau d'affections et de capacités qui le singularisent - ce qu'il mange, comme il se meut; comme il se communique; où il vit; s'il est grégaire ou solitaire... - et qui est à l'origine des perspectives. Le corps est ainsi le "grand différenciateur", ce qui unit les êtres du même type dans la mesure où il les distingue des autres corps. Lieu de l'émergence de la différence, le corps est le prototype de l'objet social, instrument fondamental d'expression du sujet et en même temps objet par excellence, ce qui se donne à voir à l'autre ${ }^{72}$. La prédation incorporante est dès lors le lieu de la transformation, dont le schéma transcendantal est le cannibalisme : dans la relation entre le tueur et la victime, la mort violente et sa logique cannibale produisent une synthèse où toutes les distances s'annulent dans un point de fusion, conduisant à la transformation rituelle d'Ego. Cette métamorphose corporelle est la contrepartie amérindienne du thème européen de la conversion spirituelle.

Plusieurs temps forts sont ainsi apparus au fil de l'étude, qu'il paraît utile de résumer autour des principaux apports de l'analyse sur le questionnement suivi à propos du modèle et de la copie, et selon les trois déclinaisons choisies de l'actualisation, de l'appréhension et de l'appropriation. Les relations au modèle sont plutôt observées jusqu'autour des $\mathrm{XI}^{\mathrm{e}}$ et $\mathrm{XII}^{\mathrm{e}}$ siècles, et plutôt dans les décors monumentaux, et elles reposent, dans une proportion non négligeable, sur la référence instituée à l'antiquité, grecque comme latine: peut-être faudrait-il mieux prendre la mesure de cette référence, en comprenant qu'il s'agit avant tout d'une référence politique et, par conséquent, qu'elle induit un ensemble d'attitudes plus tournées vers l'idée de " classique », que vers l'antique tout court. C'est aussi ce que Valentino Pace entendait par le terme qu'il utilisait d'« antiquisme » courant, selon lui, à cette époque et depuis au moins le $\mathrm{IX}^{\mathrm{e}}$ siècle, dans la koinè méditerranéenne. En revanche, et de manière paradoxale, l'importation des icônes, c'est-à-dire leur circulation sur les deux rives de la Méditerranée, montre le fait inverse : l'éloignement du modèle, la présence de la copie, et même de la copie de la copie, qui affirme la présence d'autant plus forte de l'image ${ }^{73}$. Et la conception de la présence reste étrangement grecque, signal des rencontres et des croisements intervenus, bref indice des métissages réalisés. Enfin, comment ne pas voir, dans les exemples pris en compte, entre modèle et copie, puis de la copie à la copie, et entre les images, les jeux de regards échangés, parfois sans partage, entre le même et l'autre, dans des rapports que les anthropologues, et en particulier Eduardo Viveiros de Castro, ont placés sous le signe vivifiant de la " prédation », de voir également ce que la circulation des objets permet à l'historien, et à l'historien de l'art, de reconstituer du « repensement » continuel de l'image par l'art, et inversement : toujours en mouvement, « une ontologie de l'actualité » fondée sur le passé jugé le plus authentique ${ }^{74}$. Et dans ces allers-retours, durant la chronologie du Moyen Âge, et par-delà, ce que la question du modèle et de la copie manifeste le mieux reste, sans aucun doute, la double faillite des déterminismes et des causalités tranchées : un univers de relations. 


\section{NOTES}

1. E. MAGnANiet D. RUSSO, «Histoire de l'art et anthropologie, 4. Modèle et copie. Autour de la notion de "modèle" en anthropologie, histoire et histoire de l'art ", Bucema, 14 (2010), p. 209-233 [http://cem.revues.org/index11558.html].

2. E. VIVEIROS DE CASTRO, A inconstância da alma selvagem e outros ensaios de antropologia, São Paulo, 2002 ; ID., Métaphysiques cannibales. Lignes d'anthropologie post-structurale, trad. portugais (Brésil) O. BONILLA, Paris, 2009. Voir sur ce questionnement, P. DEscola (coord.), La fabrique des images. Visions du monde et formes de la représentation, Paris, 2010 ; ID., Par-delà nature et culture, Paris, 2005.

3. Pour la position d'une problématique d'étude, qui reste à discuter toujours, depuis sa date de parution, voir H. BELTING, Image et culte. Une histoire de l'art avant l'époque de l'art, trad. fr. F. MULLER,

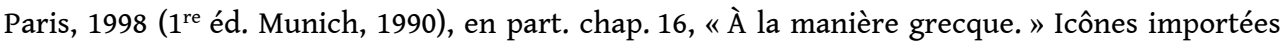
d'Orient, p. 445-472 ; ID., La vraie image. Pour une anthropologie des images, trad. fr. J. TORRENT, Paris, 2004 ; D. RUSSo, «Imago Pietatis. Une image paradoxale sur les deux rives de la Méditerranée, XIII $\mathrm{XV}^{\mathrm{e}}$ siècles », Textes, images, 5-6-7 (2008), p. 47-55. Pour une problématique centrée sur l'image et sur les études des décennies 1990-2000, K. MOXEY, "Les études visuelles et le tournant iconique », Intermédialités, 11 (2008), p. 149-168.

4. Dans un autre champ d'interrogations, celui des mathématiques, A. B ADIOU, Le concept de modèle, Paris, 2007, en part. chap. 9 et 10, p.123-134 et 135-149 (« La catégorie de modèle et l'expérimentation mathématique "; "La catégorie de modèle et le temps historique de la production mathématique »).

5. M. CARRUTHERS, Machina Memorialis. Méditation, rhétorique et fabrication des images au Moyen Âge, trad. fr. F. DURAND-BOGAERT, Paris, 2002, en part. p. 42, sur les «fables inventoriantes». Sur la notion même de «fable», A. CHASTEL, Fables, formes, figures, 3 vol., Paris, 1978, passim. Pour la notion de tradition, ici utilisée, R. KLIBANSKY, La philosophie et la mémoire du siècle. Entretiens avec Georges Leroux, Paris, 1998, en part. chap. 8, p. 195-206 ("La tradition platonicienne »).

6. Le « chemin du soleil » désigne la ceinture zodiacale dans la série de tablettes dites MOUL.APIN qui seraient datées de la fin du $\mathrm{II}^{\mathrm{e}}$ millénaire.

7. ARATos, Phénomènes, t. 1 et 2, trad. et com. J. MARTin, Paris, 1998.

8. ÉratosthèNe, Les Catastérismes. Le ciel, mythe et histoire des constellations, trad., prés. et com.

P. CHARVET et A. ZUCKER, Paris, 1998.

9. Sur la question de la mythologie dans l'art médiéval voir, notamment, E. PANOFSKY et F. SAXL, La mythologie classique dans l'art médiéval, trad. fr., Paris, 1990.

10. ARATOS, Phénomènes..., op. cit., v. 545-546.

11. H. STERN, Le calendrier de 354. Étude sur son texte et ses illustrations, Paris, 1953 (Bibliothèque archéologique et historique, 55).

12. Pour un aperçu sur la variété des occurrences zodiacales, cf. H. G. G UNDEL, Zodiakos. Tierkreisbilder im Altertum. Kosmishe Bezüg und Jenseitsvorstellungen im antiken Alltagsleben, Mayence, 1992.

13. Pour une introduction à l'organisation des images zodiacales, cf.F. G URY, «Principes de composition de l'image zodiacale », Latomus. Revue d'études latines, 53/3 (1994), p. 528-542.

14. Discussion des concepts de réseau, nexus, et de dispositif dans A. GeLL, L'art et ses agents, une théorie anthropologique, trad. fr. S. et O. RENAUT, Dijon, 2009, en part. chap. 2 et 3, p. 15-34 et 35-62 : «La théorie du réseau de l'art (Art Nexus) »; « Le réseau de l'art (Art Nexus) et l'indice ».

15. Comme par exemple, sur le pavement de mosaïque retrouvé dans une villa romaine de Münster-Sarnsheim, 270 apr. J.-C., et conservé au Landesmuseum de Bonn, montrant Helios et son quadrige entouré du cercle du zodiaque. 
16. Sur ces mosaïques disposées en pavements, cf. R. HACHLILI, Ancient mosaic pavements. Themes, issues, and trends, Leiden/Boston, 2009.

17. Pour l'expression utilisée, cf. S. GruZINSKI, La pensée métisse, Paris, 1999 ; ID. (coord.), Planète métisse, Paris, 2008, en part. p. 16-25, 32-49 et 58-71: S. GRUZINSKI, "Planète métisse ou comment parler du métissage »; C. BERNAND, « Regards d'anthropologue sur l'ambiguïté des mélanges »; S. GRUZINSKI, « Mondialisations et métissages ».

18. P. Descola, Par-delà..., op. cit., p. 183-337 ("Les dispositions de l'être ») ; ID., La fabrique des images..., op. cit., p. 11-19 (Introduction, « Manières de voir, manières de faire »).

19. P. Descola, La fabrique des images..., ibid., p. 17.

20. ISIDORE DE SEVILLE, Traité de la nature, XXVI, «Des noms des astres ", éd. J. FonTAINE; issu de sa thèse, Isidore de Séville et la culture classique dans l'Espagne wisigothique, Bordeaux, 1960 (Bibliothèque de l'École des hautes études hispaniques, 28). Pour les étymologies, voir, notamment: J.-P.GuILLAUMin, «Quelques caractéristiques de l'entreprise encyclopédique d'Isidore de Séville dans les Étymologies ", in M. BALARD etM. SOT (dir.), Au Moyen Âge, entre tradition et innovation, Paris, 2009, p. 19-33. Voir aussi pour un cadre général d'interprétation : H. INGLEBERT, Interpretatio christiana. Les mutations des savoirs (cosmographie, géographie, ethnographie, histoire) dans l'Antiquité chrétienne, Paris, 2001.

21. Pour cette notion, cf. R. KRAUTHeIMER, Idéologie de l'art antique, du IV au Xve siècle, trad. fr., Paris, 1995.

22. Par exemple, le Martyrologe de Wandalbert [CITTÀ DEL VATICANo, Bibliotheca Apostolica Vaticana, Reg. Lat. 438, IX ${ }^{\mathrm{e}}$ siècle], dans lequel chacun des mois est présenté sous une arcature par une figure exposant, comme «mettant en indice » selon le vocabulaire d'Alfred Gell, l'occcupation du mois, ainsi que par le signe du zodiaque correspondant; le Martyrologium d'Usuardus [DIJoN, Bibliothèque municipale, ms. 0634, av. 1142], où les signes du zodiaque sont peints en marge de chaque mois.

23. Par exemple, sur les plaques d'ivoire du reliquaire provenant probablement de la cathédrale de Bamberg, daté du $3^{\mathrm{e}}$ quart du $\mathrm{x}^{\mathrm{e}}$ siècle, et conservé au Bayer. Nationalmuseum de Munich (Inv. Nr. MA 175). Les signes du zodiaque sont associés aux apôtres, cf. H. G. GuNDEL, Zodiakos..., op. cit, Kat. Nr. 374, en part. p. 303 et 306. Pour cette mise en correspondance systématique, outre les ouvrages cités, cf. J. DaniéLou, Les symboles chrétiens primitifs, Paris, 1961, en part. chap. 8, p. 131-132 (« Les douze apôtres et le Zodiaque »).

24. Sur ces plans de la réflexion, cf. H. GÜNTHER, Le temps de l'histoire. Expériences du monde et catégories temporelles en philosophie de l'histoire, de saint Augustin à Pétrarque, de Dante à Rousseau, trad. fr. Paris, 1995 ; pour le monde romain et saint Augustin (354-430), cf. P. CAMBronNe, Saint Augustin, un voyage au cœur du temps, t. 2 (Les commencements), Bordeaux, 2011. Dans le premier des trois tomes de Temps et récit (Paris, $1^{\text {re }}$ éd. 1983-1985, 1991, en part. p. 158), P. Ricoeur s'interrogeait pour savoir si la question du temps humain n'aspirait pas à se résoudre par un aspect qualitatif, qu'il formulait selon des tensions graduées en ces termes: "Depuis combien de temps? Pendant combien de temps? Dans combien de temps? (...)» Nous poserons la même demande, au sujet du zodiaque, dans son inscription temporelle à l'intérieur de l'espace d'installation de certaines de ses formes, de ses figures, de ses fables, dans les ensembles monumentaux sculptés et peints.

25. Voir les réflexions dans T. F. MATHEWs, The Clash of Gods : A Reinterpretation of Early Christian Art, Princeton/Oxford, 2003 (1 $1^{\text {re }}$ éd. 1993).

26. R. HACHLILI, Ancient Mosaic..., op. cit.

27. M. Avi-YONAH, « Mosaic Pavement at El-Hammam », in Quarterly of the Department of Antiquities in Palestine, V, Beisan, 1936, p. 11-30.

28. In 15, 1-17 ; Mt 21, 33-41 ; J. DANIELoU, Les symboles..., op. cit., p. 33-48 (« La vigne et l'Arbre de Vie »). 
29. hiPpolyte DE ROME, Commentaire sur Daniel, I, 17, éd. et trad. M. LefèVRE, Paris, 1947 (Sources chrétiennes).

30. G. SAURON, «Le message symbolique des rinceaux de l'Ara Pacis Augustae ", Comptes rendus des séances de l'académie des inscriptions et Belles Lettres, 126 (1982), p. 81-101 [http://www.persee.fr/ web/revues/home/prescript/article/crai_0065-0536_1982_num_126_1_13914].

31. Voir le médaillon frappé à Rome en 326 pour commémorer les vingt ans de règne de Constantin et la légende : "Gloria saeculi Virtus Caess » («Gloire du siècle, valeur des Césars »); F. LECOCQ, «L'Empereur romain et le Phénix », in S. FABRIZIO-COSTA (éd.), Phénix : mythe(s) et signe(s), Berne, 2001, p. 27-56.

32. Ce sarcophage est actuellement conservé dans le vestibule de Saint-Laurent-hors-les-Murs de Rome.

33. Cet autel a d'abord rejoint l'église de S. Maria in Portico de Rome avant d'occuper sa place actuelle. Voir à ce sujet : D. MAzzoLENI, «L'iscrizione medievale del cippo romano », in Giornata di studio su Santa Galla, Rome, 1991, p. 55-77 et S. RiccionI, « Gli altari di S. Galla e S. Pantaleo », ibid., p. 191-193 repris brièvement dans S. RiccionI, «Litterae et figurae. Pour un art rhétorique dans la Rome de la Réforme grégorienne ", in S. ROMANO et J. ENCKELL JULLIARD (dir.), Roma e la Riforma gregoriana. Tradizioni e innovazioni artistiche (XI-XII secolo), Rome, 2007, p. 141-163.

34. F. B ISCONTI, "Un fenomeno dell "economia del reimpiego" : l'ara funeraria romana usata come altare nell'antica basilica di S. Maria in Portico ", in Giornata di studio..., ibid., p. 33-53.

35. Sancti Petri Damiani Sermones, éd. G. LUCCHESI, Turnhout, 1983 ; PETER DAMIAN, Letters, 1-180, trad. O. J. BLum, Washington D. C, 1989-2005 [De Gloria Paradisi, éd. PL, 111, col. 970-990; De bono status religiosi et tropologia variarum animantium, éd. PL, 145, col. 763-792].

36. Voir notamment: M. CARRUTHERS, Machina memorialis..., op. cit. et C. KLAPISCH-ZUBER, L'ombre des ancêtres. Essai sur l'imaginaire médiéval de la parenté, Paris, 2000.

37. BRUNO DE SEGNI, S. Brunonis Astensis abbatis montis casini et episcopi signiensium. Opera omnia, aucta et adnotationibus illustrata juxta editionem Romae anno 1791 curante Bruno Bruni datam. Accedit Oddonis Astensis... Expositio in Psalmos, S. Brunoni ab ipso auctore dicata quam ad calcem operum S. Brunonis edidit D. Maurus Marchesius... Venetiis anno 1651, Turnhout, 1970 [Sententiae, I, De figuris ecclesiae, VIII, De Evangeliis, éd. PL, 165, col. 898D-902B].

38. S. R ICcionI, «La décoration monumentale à Rome aux $\mathrm{XI}^{\mathrm{e}}$ et $\mathrm{XII}^{\mathrm{e}}$ siècles: révisions chronologiques, stylistiques et thématiques ", Perspectives. Revue de l'INHA, 2 (2010), p. 319-360.

39. J.-C. BonNE, « De l'ornement à l'ornementalité. La mosaïque absidiale de San Clemente de Rome ", in L'ornement dans la peinture murale du Moyen Âge, Poitiers, 1997, p. 103-119 et H. TOUBERT, "Le renouveau paléochrétien à Rome au début du $\mathrm{XII}^{\mathrm{e}}$ ", Cahiers archéologiques, 20 (1970), p. 99-154; EAD., Un art dirigé. Réforme grégorienne et iconographie, Paris, 1990, en part. p. 239-310.

40. J. THIRION, "Saint-Trophime d'Arles ", Congrès archéologiques de France, 134e session (1976, Pays d'Arles), Paris, 1979, p. 460-469 ; A. HARTMANN-VIRnich, «La façade de Saint-Gilles-du-Gard», Congrès archéologiques de France, 157e session (1999, Gard), Paris, 2000, p. 255-277 ; D. RIGAUX, « Pour la gloire de Dieu et le Salut des hommes, le programme iconographique du portail de SaintTrophime ", in A. HARTMANN-VIRNICH (dir.), Le portail de Saint-Trophime d'Arles, naissance et renaissance d'un chef-d'œuvre roman, Luçon, 1999, p. 25 sqq.

41. A. DE BAYNAST, «Les Mages comme figure de la conversion dans l'Antiquité tardive », Graphé, 20 (2011), p. 39-57.

42. PARIS, BnF, lat. 9428 , fol. $34 \mathrm{v}$.

43. Plat de reliure postérieure de l'évangéliaire de Lorsch: CITTÀ DEL VATICANO, Bibliotheca Apostolica Vaticana, Museo. Cristiano.

44. Cassette de l'école de Metz, dernier tiers du IX $X^{e}$ siècle, musée du Louvre.

45. SAINT AMBROISE, Exhortatio virginitatis, 4, 27, éd. PL, 16, col. 359A. 
46. $E z$ 44, 1-2: «Il me ramena vers le porche extérieur du sanctuaire, face à l'orient. Il était fermé. Yahvé me dit : ce porche sera fermé. On ne l'ouvrira pas, on n'y passera pas, car Yahvé, le Dieu d'Israël, y est passé. Aussi sera-t-il fermé. » C'est sur cette prophétie d'Ézéchiel que se construit l'exégèse comparant la Vierge à la porte close du temple.

47. MÜNCHEN, Bayerische Staatsbibliothek, Clm 4453, fol. 29r.

48. G. SCHILLER, Ikonographie der christlichen Kunst, t. 1 (Inkarnation, Kindheit, Taufe, Versuchung, Verklärung, Wirken und Wunder Christi), Gütersloh, 1966, p. 105-107. L'auteur observe le statut impérial nouveau de la Vierge à l'enfant, sans toutefois le mettre en rapport avec Hérode.

49. ANSELM De CANTERBURY, Orationes, 55, éd. PL, 158, 961C: " $O$ beata Dei genitrix, Virgo Maria, templum Dei vivi, aula Regis cecterni, sacratarium Spiritus sancti. »

50. ANSELM DE CANTERBURY, Orationes..., ibid., 962B : « O regina et domina mundi, scala cœli, tronus Dei, janua paradisi. "

51. Deux catalogues d'expositions importants, parmi bien d'autres: Holy Image. Hallowed Ground. Icons from Sinaï, The J. Paul Getty Museum (14 novembre 2006-10 mars 2007), Los Angeles, 2006, $3^{\mathrm{e}}$ éd. 2007, trois principales entrées : « Holy Image » ; « Holy Space »; « Holy Site »; Le Mont Athos et l'Empire byzantin. Trésors de la sainte Montagne, Petit Palais, musée des Beaux-Arts de la ville de Paris (10 avril-5 juillet 2009), Paris, 2009.

52. Le chapitre s'insère entre deux autres nœuds du raisonnement, d'une part «L'icône dans la vie urbaine de Rome" (chap.15) et "Norme et liberté : icônes italiennes de l'époque des communes» (chap. 17), qui le mettent en mouvement, donc en situation, dans l'histoire des communes italiennes et des villes en relations commerciales avec elles.

53. H. MAGUiRE, Art and Eloquence in Byzantium, Princeton, 1981.

54. Voir Histoire de l'art et anthropologie, Paris, 2009, en part. P. CORDEZ, « Entre histoire de l'art et anthropologie : objets et musées » [http://actesbranly.revues.org/199].

55. Voir les riches approches développées dans M.ESPAGNE, Les transferts culturels francoallemands, Paris, 1999 et Le creuset allemand. Histoire interculturelle de la Saxe, XVIII ${ }^{e}$-XIX ${ }^{e}$ siècles, Paris, 2000, autour du concept d'interculturalité et à partir de la notion de «transferts ». Pour une application à l'histoire de l'art: ID., L'histoire de l'art comme transfert culturel. L'itinéraire d'Anton Springer, Paris, 2009. Voir aussi Interactions et transferts artistiques, $\mathrm{n}^{\circ}$ spéc., Histoire de l'art 64 (2009).

56. Pour la discussion de ces concepts, cf. A. A PPADURAI, «Commodities and the Politics of Value ", in ID. (dir.), The Social Life of Things. Commodities in Cultural Perspective, Cambridge, 1986 ; voir aussi ID., Après le colonialisme. Les conséquences culturelles de la globalisation, trad. fr. F. Bouillot,

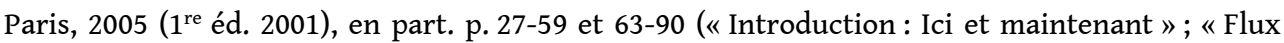
globaux »; chap. 1 « Disjonction et différence dans l'économie culturelle globale »).

57. Pour les métissages de l'image, cf. S. GRUZINSKI, La pensée métisse..., op. cit. et ID., Planète métisse..., op. cit. Sur ce point et à propos de La guerre des images (Paris, 1990), du même auteur, discussion par R. Hadj-Moussa, Anthropologie et sociétés, 16/1 (1992), en part. p. 118-120 [http:// id.erudit.org/iderudit/015206ar]. Sur le concept d'hybridation, développements dans C. BERNAND, «Regards d'anthropologue... », op. cit. et A. Russo, « À travers l'image. Invention et fabrique des métissages ", in Planète métisse..., op. cit., p. 32-49 et 90-105. Dans un autre champ géographique d'observations, cf. C. BERNAND, Un Inca platonicien. Garcilaso de la Vega, 1569-1616, Paris, 2006.

58. Présentation d'ensemble dans R. CASSANELLI (dir.), La Méditerranée des croisades, Paris, 2000 ; études de cas et problématique, D. Russo, « Art médiéval de la Méditerranée ", n spéc., Revue de l'Art, 158/4 (2007).

59. H. Belting, Image et culte..., op. cit., p. 426-427 (« Une ancienne icône dans un nouveau rôle : la Madone en avocate des Romains ») ; sur ce « rôle » de Marie, cf. G. P. Wolf, « Salus Populi Romani ». Studien zur Geschichte des römischen Kultbildes im Mittelalter, Heidelberg, 1990.

60. H. Belting, Image et culte..., ibid., p. 431-432 (fig. 188). 
61. H. Belting, Image et culte..., ibid., p. 103-104 (fig. 23). L'auteur donne pour la mesure de la tête de Marie la mention de $53 \mathrm{~cm}$. Sur le transfert de l'icône de Santa Maria Antiqua à Santa Maria Nova et ses circonstances historiques, ainsi que pour les différentes étapes de sa restauration, voir P. AMATO, «De vera effigie Mariae ». Antiche Icone romane, Rome, 1988. Il est à noter que, dès 735, l'ancienne image, imago antiqua, fut entourée d'une monture d'argent, offerte par le pape Grégoire III (731-741) ; P. AMATO, « De vera effigie Mariae »..., ibid., p. 18 ; H. BELTing, Image et culte..., ibid., p. 103, sans mention du pape, ni précision sur son action en faveur des images et contre Léon III (empereur de Byzance, de 717 à 741), durant l'iconoclastie. Sur l'insertion de l'icône dans la procession romaine avec aussi l'icône du Latran, cf. H. Belting, Image et culte..., ibid., p. 667-672, Annexe 4A-4I. H. Belting reprend [p. 103, n. 76] les données fournies par E. Kitzinger [" A Virgin's Face. Antiquarianism in Twelfth Century Art», Art Bulletin, 62 (1980), p. 6 sq.] sur la mise en rapport entre l'icône et la procession romaine de Maria Assunta. Pour l'histoire du processus reliant l'icône et la liturgie dans la vie des grands « Titres" romains, cf. W. TRonzo, « Between Icon and the Monumental Decoration of a Church », in Icon, Baltimore, 1988, p. 36 sq., et remise en situation générale par W. JACOBSEN, «L'Église du haut Moyen Âge : parcours et fonctions », in P. Piva (dir.), Art médiéval. Les voies de l'espace liturgique, Paris, 2010, p. 47-79.

62. H. Belting, Image et culte..., ibid., p. 422 et n. 10 ; V. PACE, «Presenze e influenze cipriote nella pittura duecentesca italiana », Corsi di cultura sull'arte ravenate e bizantina, 32 (1985), p. 265-266.

63. L. MOREAU DE BELLAING, La légitimation. Approche psychanalytique, sociologique et anthropologique, Paris, 1997, 3 partie, p. 145-154 et 155-169, chap. 6-7 (« Le statut de la conscience et la définition du politique dans une perspective psychanalytique »; "De l'autorité à l'obligation »). Pour une évaluation des retombées possibles sur le champ du visuel, cf. S. TISSERON, Psychanalyse de l'image. Des premiers traits au virtuel, Paris, 2010 ( $1^{\text {re }}$ éd. 1995).

64. Sur le foyer de création que fut Saint-Jean-d'Acre, entre 1250 et 1291, au carrefour des voies maritimes et terrestres, cf. É. MARASZAK, «Entre Est et Ouest, les manuscrits de Terre sainte au XIII ${ }^{\mathrm{e}}$ siècle ", in E. MAGNANI et D. RUSso, « Histoire de l'art et anthropologie... », op. cit., p. 225-233 ; pour des exemples présentés sur les villes italiennes à gouvernement communal, cf. H. BeLting, Image et culte..., op. cit., p. 473-510, et 511-552, chap. 17 et 18 (« Norme et liberté : icônes italiennes de l'époque des communes"; «Les Madones de Sienne. L'image dans la vie quotidienne et religieuse »).

65. Voir l'exemple de l'icône de la Vierge à la lettre d'intercession, cathédrale de Spolète, vers 1100 ( $31 \mathrm{~cm}$ x $24 \mathrm{~cm}$ ), entre autres ; H. BelTing, Image et culte..., ibid., p. 327 et n. 71 (sur l'icône), p. 326 (fig. 149), p. 437-439 (sur la conjoncture historique de l'actualisation de l'icône à Spolète et son " adoption » par la commune).

66. Sur l'icône de Spolète, Hans Belting note que le support de toile fut découpé en haut et en bas, quand une dame, de la famille Petralipha (?), du nom d'Irina, entra en sa possession et se préoccupa de la faire enchâsser dans du métal (cf. Image et culte..., ibid., p. 378). L'épisode, très important pour notre argumentation, serait encore à préciser sur les plans chronologique et, plus largement, historique. Pour un exemple de même type, mais dans un milieu de cour, voir celui de l'Icône de Breznice, une Vierge à l'enfant, datée de 1396 et conservée à Prague, dans la Galerie nationale de la ville ; H. Belting, Image et culte..., ibid., p. 454 (fig. 204) et 453-457.

67. Dans ce travail en cours, un guide très utile : J. Goody, La raison graphique. La domestication de la pensée sauvage, 1977, trad. fr. J. BAZIN et A. BENSA, Paris, 1978, et La peur des représentations: l'ambivalence à l'égard des images, du théâtre, de la fiction, des reliques et de la sexualité, 1997, trad. fr. P.-E. DAUZAT, Paris, 2006 ( $1^{\text {re }}$ éd. 2003). Du même auteur et pour un élargissement des perspectives : Le vol de l'histoire. Comment l'Europe a imposé le récit de son passé au reste du monde, 2006 , trad. fr. F. DURAND-BOGAERT, Paris, 2010.

68. Ce qui suit se fonde sur le recueil d'articles paru en 2002 et l'ouvrage de 2009 : E. VIVEIROS DE CASTRO, A inconstância..., op.cit.; ID., Métaphysiques cannibales..., op.cit. Voir aussi son travail de 
terrain sur les Indiens Araweté : Araweté, os deuses canibais, Rio de Janeiro, 1986, rééd. anglais From the enemy's point of view : humanity and divinity in an Amazonian society, trad. C. HOWARD, Chicago, 1992.

69. M. STRATHERN, The gender of the gift : problems with women and problems with society in Melanesia, Berkeley, 1988.

70. Cf. E. viVEIROS DE CASTRO, «Imagens da natureza e da sociedade ", in ID., A inconstância..., ibid., p. 319-344 (article de 1996).

71. Le livre Métaphysiques cannibales (op.cit.), issu d'une série de conférences réalisées par Eduardo Viveiros de Castro à Paris en janvier 2009, comme une alternative à la théorie de Philippe Descola (voir p. 48-51). Les deux auteurs ont réalisé un débat public le 30 janvier 2009, dont l'enregistrement peut être consulté dans http://www.archivesaudiovisuelles.fr/FR/ _video.asp?format=68\&id=1664\&ress=6193\&video $=131085$. Voir aussi l'éditorial de B. Latour : «Perspectivism : "type" or "bomb" ?", Anthropology Today, 25/2 (2009), p. 1-2.

72. S. BRETON (dir.),Qu'est-ce qu'un corps? Afrique de l'Ouest, Europe occidentale, Nouvelle-Guinée, Amazonie, Paris, 2006, en part. « Un corps fait de regards. Amazonie », p. 148-199.

73. Voir des remarques pertinentes dans R. RECHT, Point de fuite. Les images des images des images, Paris, 2009, en part. p. 17-74, $1^{\text {re }}$ partie «Critique et histoire de l'art».

74. En reprenant l'expression à G. Vattimo, pour qui il s'agit du mouvement même de l'histoire, ainsi qu'il le développe dans "Une ontologie de l'actualité», in ID. (dir.), Que peut faire la philosophie de son histoire?, 1988, trad. fr. C. ALunNi et alii, Paris, 1989, p. 163-186.

\section{INDEX}

Mots-clés : anthropologie, histoire de l'art 